\title{
Computing general-relativistic effects from Newtonian N-body simulations: Frame dragging in the post-Friedmann approach
}

\author{
Marco Bruni, ${ }^{*}$ Daniel B. Thomas, ${ }^{\dagger}$ and David Wands \\ Institute of Cosmology and Gravitation, University of Portsmouth, Dennis Sciama Building, \\ Burnaby Road, Portsmouth PO1 3FX, United Kingdom
}

(Received 18 June 2013; published 19 February 2014)

\begin{abstract}
We present the first calculation of an intrinsically relativistic quantity, the leading-order correction to Newtonian theory, in fully nonlinear cosmological large-scale structure studies. Traditionally, nonlinear structure formation in standard $\Lambda \mathrm{CDM}$ cosmology is studied using N-body simulations, based on Newtonian gravitational dynamics on an expanding background. When one derives the Newtonian regime in a way that is a consistent approximation to the Einstein equations, the first relativistic correction to the usual Newtonian scalar potential is a gravitomagnetic vector potential, giving rise to frame dragging. At leading order, this vector potential does not affect the matter dynamics, thus it can be computed from Newtonian N-body simulations. We explain how we compute the vector potential from simulations in $\Lambda \mathrm{CDM}$ and examine its magnitude relative to the scalar potential, finding that the power spectrum of the vector potential is of the order $10^{-5}$ times the scalar power spectrum over the range of nonlinear scales we consider. On these scales the vector potential is up to two orders of magnitudes larger than the value predicted by second-order perturbation theory extrapolated to the same scales. We also discuss some possible observable effects and future developments.
\end{abstract}

DOI: 10.1103/PhysRevD.89.044010

PACS numbers: 04.25.-g, 98.80.-k

\section{INTRODUCTION}

Modern cosmology is usually studied in two limits. On the largest scales, a perturbative approach is used in a general-relativistic framework. On small scales, nonlinearities are treated in a Newtonian fashion, often with the use of N-body simulations.

Few attempts have been made to go beyond the Newtonian approximation on nonlinear scales by including post-Newtonian type corrections [1-6]. However, no attempt has been made to include post-Newtonian corrections in N-body simulations of cosmological large-scale structure. Investigations have been carried out into the interpretation of N-body simulations on large scales, of the order of the Hubble length [7,8]. In [8], they examine the dictionary between Newtonian and relativistic cosmologies on all scales and how accurately Newtonian cosmology satisfy the Einstein equations. Of course, no matter how well the Newtonian dynamics capture the full general-relativistic (GR) dynamics, there are GR quantities on all scales that have no counterpart in Newtonian theory.

Recently, a new approximation scheme has been developed, dubbed the post-Friedmann approach [9], with the aim of providing a unified framework for all scales, from the fully nonlinear Newtonian regime to the largest scales where relativistic effects become important $[10,11]$. It is based on an expansion in inverse powers of the speed

\footnotetext{
*marco.bruni@port.ac.uk

daniel.b.thomas@port.ac.uk

¥david.wands@port.ac.uk
}

of light, $c$, in a post-Newtonian [12] fashion, adapted to cosmology. When linearized, this approach correctly reproduces the linear general-relativistic perturbation theory. When one derives the Newtonian regime in this approach, in a way that is a consistent approximation to the Einstein equations, a vector potential must be present in the metric in addition to the usual Newtonian scalar gravitational potential.

This vector potential is nondynamical at leading order, therefore it does not affect the matter dynamics. It is sourced only by terms that appear in Newtonian gravity, so it can be extracted from N-body simulations. Physically, this vector potential represents the gravitomagnetic field that is generally present in metric theories of gravity such as general relativity. Its typical effect is frame dragging, a ubiquitous relativistic effect, well known in cosmological perturbation theory [13] and in black hole systems [14]. Furthermore, in the solar system, Gravity Probe B [15] has measured the frame dragging of the Earth, see also [16]. The computation of the frame-dragging vector potential from Newtonian N-body cosmological simulations is the main result of this paper. It is the first time that an intrinsically relativistic quantity, i.e. a quantity with no counterpart in Newtonian cosmology, has been computed in fully nonlinear cosmological large-scale structure studies, in particular using N-body simulations on cosmological scales [17].

\section{POST-FRIEDMANN APPROACH}

We briefly present the pertinent details of the postFriedmann approach [9]. The starting point is an expansion of the perturbed metric, in Poisson gauge [21-23], up to order $c^{-5}$ : 


$$
\begin{aligned}
g_{00} & =-\left[1-\frac{2 U_{N}}{c^{2}}+\frac{1}{c^{4}}\left(2 U_{N}^{2}-4 U_{P}\right)\right] \\
g_{0 i} & =-\frac{a B_{i}^{N}}{c^{3}}-\frac{a B_{i}^{P}}{c^{5}} \\
g_{i j} & =a^{2}\left[\left(1+\frac{2 V_{N}}{c^{2}}+\frac{1}{c^{4}}\left(2 V_{N}^{2}+4 V_{P}\right)\right) \delta_{i j}+\frac{h_{i j}}{c^{4}}\right] .
\end{aligned}
$$

Note that the background metric here is the flat FriedmannLemaître-Robertson-Walker (FLRW) metric, not the Minkowski metric, i.e. a standard $\Lambda \mathrm{CDM}$ cosmology is assumed. The $g_{00}$ and $g_{i j}$ scalar potentials have been split into the Newtonian $\left(U_{N}, V_{N}\right)$ and post-Friedmann $\left(U_{P}, V_{P}\right)$ components. Similarly, the vector potential has been split up into $B_{i}^{N}$ and $B_{i}^{P}$. Since this metric is in the Poisson gauge, the three-vectors $B_{i}^{N}$ and $B_{i}^{P}$ are divergenceless, $B_{i, i}^{N}=0$ and $B_{i, i}^{P}=0$. In addition, $h_{i j}$ is transverse and tracefree, $h_{i}^{i}=h_{i j}^{l}=0$. Note that at this order $h_{i j}$ is not dynamical, so it does not represent gravitational waves. From a postFriedmann viewpoint, there are two different levels of perturbations in the theory, corresponding to terms of order $c^{-2}$ and $c^{-3}$, or of order $c^{-4}$ and $c^{-5}$, respectively. Defining "resummed" variables, such as $\Phi=-U_{N}+c^{-2}\left(U_{N}^{2}-\right.$ $\left.2 U_{P}\right)$, then calculating the Einstein equations and linearizing them, reproduces linear GR perturbation theory in Poisson gauge. Thus, this approach is capable of describing structure formation on the largest scales.

By retaining only the leading order terms in the $c^{-1}$ expansion, one recovers Newtonian cosmology, albeit with a couple of subtleties. The first is that the space-time metric is a well-defined approximate solution of the Einstein equations. The second is that we have an additional equation, which is a constraint equation for the frame-dragging vector potential $B_{i}^{N}$. Once the FLRW background has been subtracted, the full system of equations, as obtained from the Einstein and hydrodynamic equations [9,24], is as follows.

$$
\begin{aligned}
& \frac{d \delta}{d t}+\frac{v_{, i}^{i}}{a}(1+\delta)=0, \\
& \frac{d v_{i}}{d t}+\frac{\dot{a}}{a} v_{i}=\frac{1}{a} U_{N, i}, \\
& \frac{1}{c^{2} a^{2}} \nabla^{2} V_{N}=-\frac{4 \pi G}{c^{2}} \bar{\rho} \delta, \\
& \frac{2}{c^{2} a^{2}} \nabla^{2}\left(V_{N}-U_{N}\right)=0, \\
& \frac{1}{c^{3}}\left[\frac{2 \dot{a}}{a^{2}} U_{N, i}+\frac{2}{a} \dot{V}_{N, i}-\frac{1}{2 a^{2}} \nabla^{2} B_{i}^{N}\right]=\frac{8 \pi G \bar{\rho}}{c^{3}}(1+\delta) v_{i} .
\end{aligned}
$$

As expected, we have the Newtonian continuity, Euler and Poissons equation from the Einstein equations, where $\bar{\rho}$ is the background matter density and $\delta=(\rho-\bar{\rho}) / \bar{\rho}$ the density contrast. There is also an equation forcing the scalar potentials $V_{N}$ and $U_{N}$ to be equal, consistent with there being only one scalar potential in Newtonian theory. The final equation is the extra equation showing that, even in the Newtonian regime, the frame-dragging potential $B_{i}^{N}$ should not be set to zero in general; this would correspond to putting an extra constraint on the Newtonian dynamics, as is made clear by taking the curl of this equation.

The potential $B_{i}^{N}$ is sourced by the vector part of the energy current $\rho \mathbf{v}$ : Taking the curl of the vector potential equation in order to remove the scalar part, we obtain

$$
\nabla \times \nabla^{2} \mathbf{B}^{N}=-\left(16 \pi G \bar{\rho} a^{2}\right) \nabla \times[(1+\delta) \mathbf{v}] .
$$

Thus setting $B_{i}^{N}=0$ would require the $\rho \mathbf{v}$ Newtonian field to be irrotational, which clearly is not the case in the nonlinear regime (in particular, after orbit crossing [25]). Note that this equation is essentially the same as the equivalent equations in $[4,8,26]$. Although $B_{i}^{N}$ doesn't influence the matter dynamics at this order, it is part of the metric and will affect cosmological observables through its effect on photon geodesics. We discuss some of the possible observational consequences later. We now compute the right-hand side of Eq. (3) from $\mathrm{N}$-body simulations and thus construct the power spectrum of the vector potential.

We will be dealing with vector quantities, for which there are different ways to define the power spectrum. Our power spectrum for a generic vector $\mathbf{v}$ is defined via

$$
\left\langle\tilde{\mathbf{v}}(\mathbf{k}) \cdot \tilde{\mathbf{v}}^{*}\left(\mathbf{k}^{\prime}\right)\right\rangle=(2 \pi)^{3} \delta^{3}\left(\mathbf{k}-\mathbf{k}^{\prime}\right) P_{\mathbf{v}}(k) .
$$

Note that for a divergenceless vector, such as $\mathbf{B}^{N}$, $k^{2} P_{\mathbf{B}^{N}}(k)=P_{\nabla \times \mathbf{B}^{N}}(k)$. With our Fourier transform convention, the dimensionless power spectrum for a field $\mathrm{X}$ is given by $\mathcal{P}_{X}(k)=k^{3} P_{X}(k) / 2 \pi^{2}$. From Eq. (3), the power spectrum of the vector potential is given by

$$
P_{\mathbf{B}^{N}}(k)=\left(\frac{16 \pi G \bar{\rho} a^{2}}{k^{2}}\right)^{2} \frac{1}{k^{2}} P_{\delta v}(k),
$$

with

$$
\begin{aligned}
P_{\delta v}= & P_{\nabla \times \mathbf{v}}(k)+P_{\delta \nabla \times \mathbf{v}}(k)+P_{(\nabla \delta) \times \mathbf{v}}(k) \\
& +P_{(\nabla \delta \times \mathbf{v})(\nabla \times \mathbf{v})}(k)+P_{(\nabla \delta \times \mathbf{v})(\delta \nabla \times \mathbf{v})}(k) \\
& +P_{(\delta \nabla \times \mathbf{v})(\nabla \times \mathbf{v})}(k) .
\end{aligned}
$$

\section{SIMULATIONS}

We have run three N-body simulations with $N_{\text {part }}=$ $1024^{3}$ particles and length $160 h^{-1} \mathrm{Mpc}$, using Gadget-2 [27], in order to compute the vector potential, as well as multiple additional runs with varying number of particles and box size. To allow comparison to previous studies of vorticity [25] the simulations were run with dark matter particles only and with a cosmology $\Omega_{m}=0.27$, $\Omega_{\Lambda}=0.73, \Omega_{b}=0.046, h=0.72, \tau=0.088, \sigma_{8}=0.9$ and $n_{s}=1$. All of the simulations started at redshift 50 and had their initial conditions created using 2LPTic [28]. 
Traditional methods of extracting fields from N-body simulations, such as cloud-in-cells (CIC) [29] have several shortcomings when applied to velocity fields. The field is only sampled where there are particles, so in a low density region the velocity field is artificially set to zero. In addition, the extracted field will be a mass-weighted, rather than volume weighted field.

To extract the velocity field, we have used the publicly available Delauney Tessellation Field Estimator (DTFE) code [30]. This code works by first constructing the Delauney tessellation $[31,32]$ and then linearly interpolating the velocities of the nodes across each tetrahedron. The velocity field and its gradients are now known everywhere. In order to get a smoothed field from the simulation, a regu$\operatorname{lar} \mathrm{N}_{\text {grid }}^{3}$ is laid down. The code then samples points at random in each grid cell and averages the results, giving a value for each grid cell. For this analysis, the code sampled 100 points per grid cell. However, varying this up to 1000 made no difference to the results.

One of the disadvantages of the tessellation code is that, unlike CIC methods, the window function cannot be easily deconvolved; the window function will be different in different regions of the simulation. We can examine the effects of the window function by varying the grid size used to analyze a given simulation. Our main result was calculated using $\mathrm{N}_{\text {grid }}^{3}=\mathrm{N}_{\text {part }}$, but varying the grid size makes no difference except on the smallest scales.

The output from the DTFE code is Fourier transformed and the modulus-squared values of the transformed field are averaged in bins for given ranges of wave number $k$. We used $N_{\text {grid }} / 4$ bins in our analyses, however varying this value did not affect the results.

\section{CONVERGENCE AND ROBUSTNESS}

First, we consider some consistency checks on the extracted fields. We calculate the density power spectrum with the state-of-the-art code POWMES [33] and check for consistency with our DTFE result. In addition, the power spectrum of the gradient of the density, which is part of one of the quantities required for the vector potential, can be extracted by itself. The power spectrum of the gradient of the density should satisfy $P_{\nabla \delta}(k)=k^{2} P_{\delta}(k)$, so we can check that the extraction of the two fields is consistent. A similar check can be performed for the velocity fields: As pointed out by [25], $k^{2} P_{\mathbf{v}}=P_{\nabla \cdot \mathbf{v}}+P_{\nabla \times \mathbf{v}}$, so we can extract all three fields and check that they satisfy this relation. The fields do indeed satisfy this constraint, up to the smallest scales where the window function starts to have an effect. This is one way to see the effects of the window function.

We can also compare our extracted velocity spectra to [25] where the velocity spectra were also extracted using the Delauney tessellation method. However, a different code was used that implemented the tessellation differently, see [25] for details. For simulations with the same parameters, our extracted vorticity power spectra are consistent with this paper and show the same dependence on resolution.

A full study of the effect of box size and mass resolution on the extracted vector potential is beyond the scope of this work. Nonetheless, for high resolution simulations that are suitable for studies of vorticity, there appear to be no significant systematic issues with resolution or box size. However, the variation amongst realisations is greater for quantities such as the vorticity, and by extension the vector potential, than for quantities such as the density and velocity divergence. This further complicates the issue and increases the required computational resources. A comprehensive study will be presented in a forthcoming publication [34].

\section{RESULTS}

The power spectra, averaged from three high resolution $\mathrm{N}$-body simulations of length $160 h^{-1} \mathrm{Mpc}$ and $\mathrm{N}_{\text {part }}=1024^{3}$, of the three source terms of the vector potential, $\delta \nabla \times \mathbf{v}, \quad \nabla \delta \times \mathbf{v}$ and $\nabla \times \mathbf{v}$, are shown in Fig. 1 alongside the linear and nonlinear matter power spectra. The power spectra plotted here are given by $P(k) /\left(f^{2} \mathcal{H}^{2}(2 \pi)^{3}\right)$, where $\mathcal{H}$ is the conformal time Hubble constant and $f=d \ln D / d \ln a$ is the logarithmic derivative of the linear growth factor $D$. These units are chosen such that the power spectrum of the velocity divergence agrees with the density power spectrum on linear scales, following [25]. We can see that it is the nonlinear terms that are the dominant sources of the vector potential,

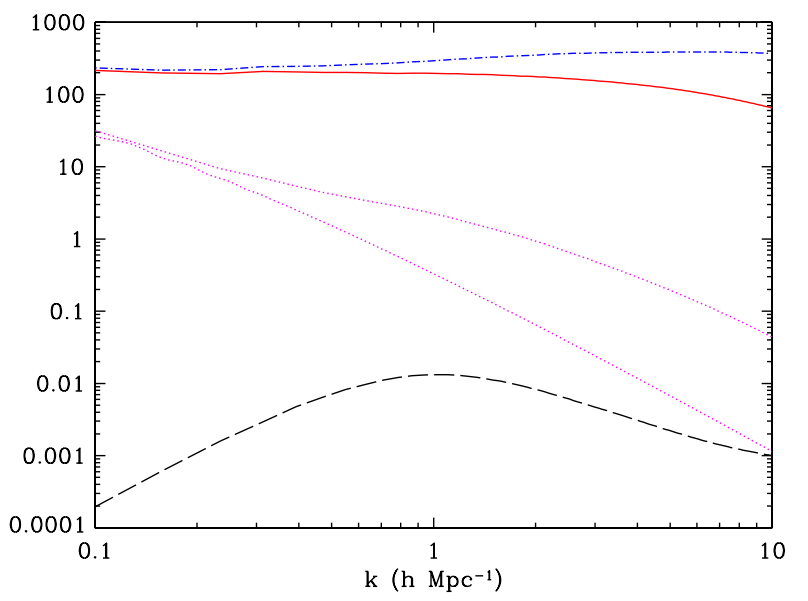

FIG. 1 (color online). The power spectra of the three source terms of the vector potential, as extracted from N-body simulations. The solid (red) line is for $\delta \nabla \times \mathbf{v}$, the dot-dashed (blue) line is for $\nabla \delta \times \mathbf{v}$, the dashed (black) line is for $\nabla \times \mathbf{v}$ and the dotted (magenta) lines are the linear and nonlinear matter power spectra for comparison. The power spectra plotted here are given by $P(k) /\left(f^{2} \mathcal{H}^{2}(2 \pi)^{3}\right)$, where $\mathcal{H}$ is the conformal time Hubble constant and $f=d \ln D / d \ln a$ is the logarithmic derivative of the linear growth factor $D$, see text and [25]. 


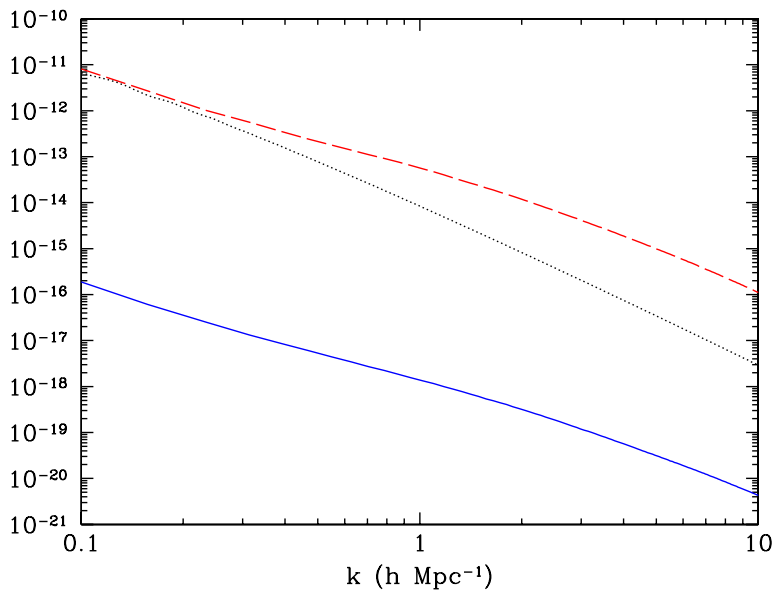

FIG. 2 (color online). The power spectra $\mathcal{P}_{\Phi}$ and $\mathcal{P}_{\mathbf{B}^{N}}$ of the Newtonian scalar potential (dashed red line) and the framedragging vector potential (solid blue line) as a function of scale, as extracted from N-body simulations. The dotted (black) line is the power spectrum of the linear theory scalar potential for comparison.

with the vorticity contribution (the sole contribution in linear perturbation theory) being subdominant on all scales.

The average of the power spectra of the vector potential over the three high resolution simulations is shown in Fig. 2, along with the average of the power spectra of the standard scalar gravitational potential. The power spectra plotted here are the dimensionless power spectra $\mathcal{P}_{\Phi}$ and $\mathcal{P}_{\mathbf{B}^{N}}$, as defined earlier, in natural units where $c=1$. For comparison, the power spectrum of the linear theory scalar potential is shown as well.

In Fig. 3 we show the ratio of the average of the vector potential power spectra to the average of the scalar potential power spectra as a function of scale. The plot shows that this ratio is fairly constant well into the nonlinear regime,

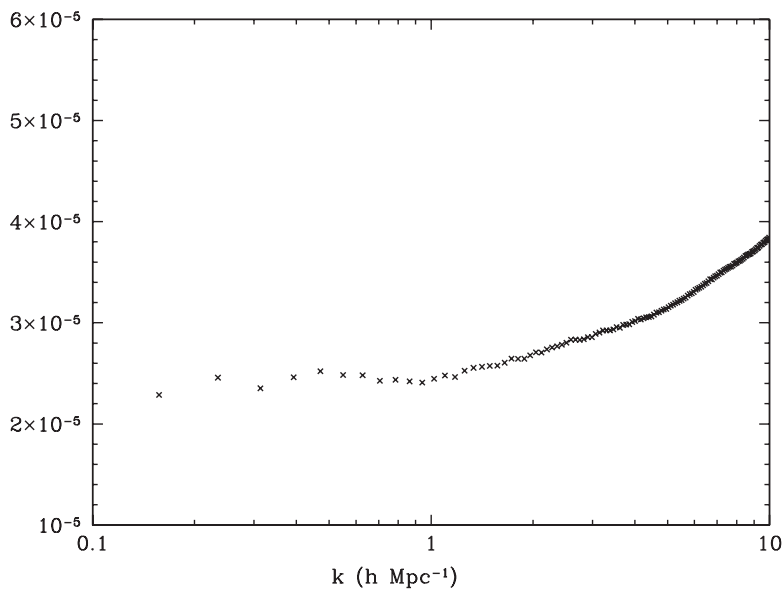

FIG. 3. The ratio of the power spectra $\mathcal{P}_{\mathbf{B}^{N}}$ and $\mathcal{P}_{\Phi}$ of the gravitomagnetic vector potential and the Newtonian scalar potential as a function of scale, as extracted from N-body simulations. with the vector power spectrum being of order $10^{5}$ times smaller than the scalar power spectrum.

In [35] (see also [36]), the vector potential at second order in general-relativistic perturbation theory is calculated. Since this method has a different regime of validity to ours, the two methods should not be expected to agree fully. However, we can compare the qualitative behavior of the two results. Over the range of overlap for the two methods, at the largest scales considered here, in both cases the ratio between the vector and scalar power spectra has a fairly constant value of order $10^{-5}$. This similar qualitative behavior is reassuring. The difference is that the vector power spectrum in [35] is of order $10^{-5}$ times the linear scalar power spectrum, whereas ours is relative to the fully nonlinear scalar power spectrum. Therefore, quantitatively our vector power spectrum is up to two orders of magnitude larger than the one from second-order perturbation theory.

\section{OBSERVABILITY}

A vector potential present in the metric will influence several cosmological observables. The most obvious one is weak gravitational lensing. The usual observable considered in weak lensing is the convergence, $\kappa$, which is the isotropic expansion of a galaxy image. Here, we show how the vector potential affects the convergence power spectrum $P_{\kappa}$. We follow a treatment similar to [37], however we explicitly include powers of $c$ and work up to order $c^{-3}$ rather than using linear general relativistic perturbation theory.

The starting point is the metric $g_{00}=-1, g_{0 i}=$ $-a c^{-3} B_{i}^{N}$ and $g_{i j}=a^{2} \delta_{i j}$. This yields a deflection angle

$$
\theta_{i}^{\prime}=\theta_{i}+\int_{0}^{\chi} \mathrm{d} \chi^{\prime}\left(B_{\chi, i}^{N}-B_{i, \chi}^{N}\right)\left(1-\frac{\chi^{\prime}}{\chi}\right)
$$

Compared to [37], the $\dot{B}_{i}^{N}$ term has vanished since it is order $c^{-4}$ in this expansion. The convergence is the trace of the distortion matrix $\psi_{i j}$, given by $\psi_{i j}=\partial \theta_{i}^{\prime} /$ $\partial \theta_{j}-\delta_{i j}$. Following [38], and working in the small angle limit with the Limber approximation, gives a convergence power spectrum

$$
P_{\kappa}^{B^{N}}(l)=\frac{l^{4}}{4} \int_{0}^{\chi_{\infty}} \mathrm{d} \chi \frac{g^{2}(\chi)}{\chi^{6}} P_{B^{N}}(l / \chi),
$$

where $g(\chi)$ is the weak-lensing weight function. The convergence power spectrum caused by the scalar gravitational potential is

$$
P_{\kappa}^{\Phi}(l)=l^{4} \int_{0}^{\chi_{\infty}} \mathrm{d} \chi \frac{g^{2}(\chi)}{\chi^{6}} P_{\Phi}(l / \chi) .
$$

We can see that the vector and scalar potentials contribute in a similar fashion to the convergence power spectrum. Therefore, because the vector power spectrum is smaller 
than the scalar power spectrum, the convergence from nonlinear scales is well approximated by just the scalar potential, as implicitly assumed in literature. However, at order $c^{-4}$, the time derivative of the vector potential will generate the odd parity B-mode of cosmic shear that is not generated by the scalar potential at first order. In forthcoming work, we will examine the weak lensing power spectra up to order $c^{-4}$ and investigate the observability of the vector potential [34].

\section{CONCLUSIONS AND DISCUSSION}

The post-Friedmann approach [9] provides a framework for examining post-Newtonian effects in cosmology. The primary result of this paper is the computation of the post-Friedmann frame-dragging vector potential at leading order, i.e. in the Newtonian dynamical regime, as shown in Fig. 2. This is the first time that an intrinsically relativistic quantity has been calculated in full nonlinearity in N-body simulations of cosmological large-scale structure formation.

For sufficiently high resolution simulations, the power spectrum of the vector potential appears to converge. The agreement of the density and vorticity fields with other methods $[33,25]$ and similar qualitative behavior of the vector potential to second-order perturbation calculations [35] support our numerical results.

Over the range of (nonlinear) scales we consider, we find that the ratio between the vector and scalar power spectra has a fairly constant value of order $10^{-5}$, similar to [35]. This ratio is much larger than the value one would naively expect from second-order perturbation theory. The difference with [35] is quantitative: on fully nonlinear scales there is more power in the scalar power spectrum, and correspondingly in the generated vector power spectrum, which is up to two orders of magnitude larger than the one from second-order perturbation theory.

As mentioned above, although this vector potential does not influence matter dynamics at leading order, it will affect photon geodesics, so the first place to look for the effects of this vector potential is in weak-lensing surveys. The small ratio between the power spectra of the vector gravitational potential and the scalar gravitational potential means that the effects of the vector potential are unlikely to be detected in the usual convergence or E-mode spectra. However, the time derivative of a vector potential generates the B-mode spectrum [37], which is not produced by the scalar potential and thus may allow the vector potential to be observed. Another effect where the vector potential may be observable is lensing of the cosmic microwave background, particularly polarization.

The magnitude of the vector potential we have computed also supports the validity of Newtonian N-body simulations in $\Lambda$ CDM cosmology: Since the vector potential is the first relativistic addition to Newtonian theory, its small magnitude relative to the scalar potential supports the assertion that on sufficiently small scales, the relativistic corrections to Newtonian gravity are sufficiently subdominant. A much larger measured value would suggest that a relativistic treatment is essential for structure formation in $\Lambda \mathrm{CDM}$. From the point of view of [8], the small size of the vector potential suggests that the abridged dictionary, corresponding to the dictionary in [7], can be used, at least on sufficiently small scales. Nonetheless, as shown here, even in a regime where the cosmological dynamics is Newtonian a relativistic framework is essential for the interpretation, and relativistic effects can be computed that are potentially observable. At next order, $c^{-4}$, the nonzero difference between the two scalar potentials that appear in the metric (1) in the post-Friedmann approach $[9,24]$, consistently with second-order relativistic perturbation theory $[22,23]$ and other studies [8], can also be computed from purely Newtonian nonlinear source terms, and its effects remain to be studied. These relativistic nonlinear effects are potentially more important in clustering/coupled dark energy and modified gravity cosmological models.

\section{ACKNOWLEDGMENTS}

We thank Marius Cautun for help with the DTFE code. The simulations in this paper were run on the SCIAMA computer at Portsmouth and analysed on COSMOS at Cambridge. This work was supported by STFC Grants No. ST/H002774/1, No. ST/K00090X/1, and No. ST/ L005573/1. Please contact the authors to request access to research materials discussed in this paper.
[1] K. Tomita, Prog. Theor. Phys. 85, 1041 (1991).

[2] M. Shibata and H. Asada, Prog. Theor. Phys. 94, 11 (1995).

[3] S. Matarrese and D. Terranova, Mon. Not. R. Astron. Soc. 283, 400 (1996).

[4] M. Takada and T. Futamase, Mon. Not. R. Astron. Soc. 306, 64 (1999).
[5] C. Carbone and S. Matarrese, Phys. Rev. D 71, 043508 (2005).

[6] J.-c. Hwang, H. Noh, and D. Puetzfeld, J. Cosmol. Astropart. Phys. 03 (2008) 010.

[7] N. E. Chisari and M. Zaldarriaga, Phys. Rev. D 83, 123505 (2011). 
[8] S. R. Green and R. M. Wald, Phys. Rev. D 85, 063512 (2012).

[9] I. Milillo, D. Bertacca, M. Bruni, and A. Maselli (to be published).

[10] M. Bruni, R. Crittenden, K. Koyama, R. Maartens, C. Pitrou, and D. Wands, Phys. Rev. D 85, 041301 (2012).

[11] M. Bruni, J. C. Hidalgo, N. Meures, and D. Wands, Astrophys. J. (in press).

[12] S. Chandrasekhar, Astrophys. J. 142, 1488 (1965).

[13] J. M. Bardeen, Phys. Rev. D 22, 1882 (1980).

[14] R. Owen et al, Phys. Rev. Lett. 106, 151101 (2011).

[15] C. W. F. Everitt et al, Phys. Rev. Lett. 106, 221101 (2011).

[16] I. Ciufolini and E. C. Pavlis, Nature (London) 431, 958 (2004).

[17] There are computations of gravitational waves produced by halo formation using a triaxial collapse model [18], and by galaxy mergers using $\mathrm{N}$-body simulations on galactic scales using specific initial conditions [19,20], but our vector potential is directly extracted from the fully nonlinear cosmological density field as computed from $\mathrm{N}$-body simulations on cosmological scales using standard initial conditions. Gravitational waves are subleading with respect to the vector potential, see below.

[18] C. Carbone, C. Baccigalupi, and S. Matarrese, Phys. Rev. D 73, 063503 (2006).

[19] V. Quilis, A. C. González-García, D. Saez, and J. A. Font, Phys. Rev. D 75, 104008 (2007).

[20] T. Inagaki, K. Takahashi, S. Masaki, and N. Sugiyama, Phys. Rev. D 82, 124007 (2010).

[21] C.-P. Ma and E. Bertschinger, Astrophys. J. 455, 7 (1995).
[22] S. Matarrese, S. Mollerach, and M. Bruni, Phys. Rev. D 58, 043504 (1998).

[23] K. A. Malik and D. Wands, Phys. Rep. 475, 1 (2009).

[24] I. Milillo, Ph.D. thesis, University of Portsmouth, 2010.

[25] S. Pueblas and R. Scoccimarro, Phys. Rev. D 80, 043504 (2009).

[26] J.-c. Hwang and H. Noh, J. Cosmol. Astropart. Phys. 04 (2013) 035.

[27] V. Springel, Mon. Not. R. Astron. Soc. 364, 1105 (2005).

[28] M. Crocce, S. Pueblas, and R. Scoccimarro, Mon. Not. R. Astron. Soc. 373, 369 (2006).

[29] R. W. Hockney and J. W. Eastwood, Computer Simulation Using Particles (McGraw-Hill, New York, 1981).

[30] M. C. Cautun and R. van de Weygaert, arXiv:1105.0370.

[31] W. E. Schaap and R. van de Weygaert, Astron. Astrophys. 363, L29 (2000).

[32] R. van de Weygaert and W. Schaap, in Data Analysis in Cosmology, edited by V. J. Martínez, E. Saar, E. MartínezGonzález, and M.-J. Pons-Bordería, Lecture Notes in Physics Vol. 665 (Springer Verlag, Berlin, 2009), p. 291.

[33] S. Colombi, A. Jaffe, D. Novikov, and C. Pichon, Mon. Not. R. Astron. Soc. 393, 511 (2009).

[34] D. B. Thomas, M. Bruni, and D. Wands (to be published).

[35] T. H.-C. Lu, K. Ananda, C. Clarkson, and R. Maartens, J. Cosmol. Astropart. Phys. 02 (2009) 023.

[36] S. Mollerach, D. Harari, and S. Matarrese, Phys. Rev. D 69 , 063002 (2004).

[37] D. B. Thomas, C. R. Contaldi, and J. Magueijo, Phys. Rev. Lett. 103, 181301 (2009).

[38] S. Dodelson, Modern cosmology (Academic Press, New York, 2003). 